\title{
Combining self-organizing maps and biplot analysis to preselect maize phenotypic components based on UAV high-throughput phenotyping platform
}

Liang Han ${ }^{1,2,4 \dagger}$, Guijun Yang ${ }^{1 *+}$, Huayang Dai ${ }^{4}$, Hao Yang ${ }^{1,3}$, Bo Xu ${ }^{1}$, Heli Li ${ }^{3}$, Huiling Long ${ }^{1,3}$, Zhenhai Li ${ }^{3}$, Xiaodong Yang ${ }^{1,3}$ and Chunjiang Zhao ${ }^{1,3^{*}}$

\begin{abstract}
Background: With environmental deterioration, natural resource scarcity, and rapid population growth, mankind is facing severe global food security problems. To meet future needs, it is necessary to accelerate progress in breeding for new varieties with high yield and strong resistance. However, the traditional phenotypic screening methods have some disadvantages, such as destructive, inefficient, low-dimensional, labor-intensive and cumbersome, which seriously hinder the development of field breeding. Breeders urgently need a high-throughput technique for acquiring and evaluating phenotypic data that can efficiently screen out excellent phenotypic traits from large-scale genotype populations.

Results: In the present study, we used an unmanned aerial vehicle (UAV) high-throughput phenotyping (HTP) platform to collect RGB and multispectral images for a breeding program and acquired multiple phenotypic components (or traits), such as plant height, normalized difference vegetation index, biomass accumulation, plant-height growth rate, lodging, and leaf color. By implementing self-organizing maps and principal components analysis biplots to establish phenotypic map and similarity, we proposed an UAV-assisted HTP framework for preselecting maize (Zee mays L.) phenotypic components (or traits).

Conclusions: This framework gives breeders additional information to allow them to quickly identify and preselect plants that have genotypes conferring desirable phenotypic components out of thousands of field plots. The present study also demonstrates that remote sensing is a powerful tool with which to acquire abundant phenotypic components. By using these rich phenotypic components, breeders should be able to more effectively identify and select superior genotypes.
\end{abstract}

Keywords: SOM, Biplot, UAV, Maize, High-throughput phenotyping

\footnotetext{
*Correspondence: guijun.yang@163.com; zhaocj@nercita.org.cn

${ }^{\dagger}$ Liang Han and Guijun Yang contributed equally to this work and should

be considered co-first author

${ }^{1}$ Key Laboratory of Quantitative Remote Sensing in Agriculture

of Ministry of Agriculture, Beijing Research Center for Information

Technology in Agriculture, Beijing 100097, China

Full list of author information is available at the end of the article
} 


\section{Background}

With the imminent threat of environmental deterioration, natural resource scarcity, and rapid population growth, mankind is facing an unprecedented challenge of producing sufficient food to ensure global food security in the coming decades $[1,2]$. It is estimated that farmers must produce $70 \%$ more food by 2050 to feed a population expected to reach about 9.6 billion [3]. Improving genetic gains and maintaining a stable food supply are effective measures for plant breeders and geneticist to alleviate the current situation. In the last two decades, crop sequencing technology has developed rapidly, allowing the whole genome to be sequenced rapidly at low cost. However, because of the lack of assistant phenotypic knowledge, methods for rapid identification of desirable traits have advanced little $[4,5]$. With increasing demand for rapid phenotyping of large numbers of lines and to accelerate progress in breeding for novel traits, phenotyping is often considered the bottleneck of crop breeding [6].

Recent advance in high-throughput phenotyping (HTP) technologies has provided a positive response to narrow the gap between the wealth of genomic data with phenotypic data [7]. HTP technologies allow large numbers of plants to be measured in a non-destructive manner with accuracy and precision. Initially, high-throughput phenotyping was applied in controlled environments, such as greenhouses and growth chambers, to collect phenotypic data from model organisms [8]. This is indoor shoot-based phenotyping that have an advantage in characterizing individual plants grown in pots, and not limited by overlapping canopies and variable environmental conditions due to soil, temperature, water etc. However, the main concern for many breeders is that the complex traits obtained by using HTP technologies in controlled environments may not be fully replicated in the field, so phenotyping in field conditions remains a bottleneck that hinders advances in breeding $[5,6,9]$.

With continuous advances in proximal sensing, fieldbased HTP has become widespread in the breeding programs. Recently, several field-based HTP platforms were developed to measure phenotypic traits, including ground-based HTP platforms [10-12] and aerial-based HTP platforms [13-15]. Ground-based HTP platforms consisting of modified vehicles have the advantages of high resolution, flexible design, and large payload, but have limitations in the portability and scale at which they can be used [16]. Compared to ground-based HTP platforms, aerial-based HTP platforms enable the rapid evaluation of the populations consisting of thousands to tens of thousands of plots and the synchronized measurements of multiple traits in an efficient manner, overcoming some limitations associated with the ground-based
HTP platforms. As one of emerging technologies in aerial-based platforms, unmanned aerial vehicles (UAV) for HTP have undergone a remarkable development in recent years and been capable of gaining advantages of their portability, operability, low cost, and high spatiotemporal resolution $[13,17,18]$. UAV-HTP based on proximal remote sensing has been envisaged to bridge the gap between ground-based measurements and satellite observations [19]. Traditional ground-based phenotyping techniques are time-consuming, labor intensive and impractical for large-scale operations [20]. Despite the advantages of satellite remote sensing in large-scale observation, it remains some limitations, such as low resolution, long revisit period and high susceptibility to water vapor [21]. In conclusion, compared with other technologies, UAV-HTP offers excellent opportunities for rapid and non-destructive extraction of crop phenotypic information in the field.

Currently, several structural and physiological agronomic traits suitable for HTP have been proposed for use in breeding programs, including but not limited to the normalized difference vegetation index (NDVI) [11, 22, $23]$, biomass accumulation [21, 24], plant height [25, 26], plant-height growth rate [15, 27, 28], lodging [29, 30], leaf color [24, 31], and yield [32-34]. Previous research has demonstrated that measurements provided by HTP platforms are highly correlated with manual reference measurements [26, 34, 35]. By using HTP technologies capable of collecting phenotypic data at multiple time points or throughout the season, researchers can better understand how traits develop, allowing better optimization of genotypes through selection in breeding programs [36].

A preliminary approach with easily measurable phenotypic traits provides a chance to select genotypes [37]. Cluster and correlation analyses seem to be a promising approach for identifying potential associations between phenotype and genotype [38, 39], which clarifies gene co-expression and phenotypic similarity. Self-organizing maps (SOMs) are a type of artificial neural network invented by Kohonen [40] that are trained by using unsupervised learning to project high-dimensional, complex data onto a two-dimensional grid. This reduces dimensionality and enhances the visualization of clustering [41, 42]. A principal components analysis (PCA) biplot highlights the extent to which the objects in rows (samples) differ from the objects in columns (features) [43]. In this context, a PCA biplot shows the largest patterns in the data in terms of how the phenotypic components differ in different genotypes.

In the present study, we used a UAV HTP platform to collect RGB and multispectral (spectral bands: green, red, red-edge and near-infrared) images for a breeding program and acquired multiple phenotypic components 
(or traits). The specific objectives for this study were (i) to propose an UAV-assisted HTP framework to establish phenotypic maps and similarities, (ii) to identify selection strategies for different breeding targets or multiple phenotypic components, and (iii) to assess the potential for using UAV field-based HTP platforms for selection decisions in a large breeding program.

\section{Methods}

\section{Field trials}

Field breeding trials included a natural population and a doubled-haploid population (i.e., 800 maize plots). The natural population assessed in this study consisted of 482 maize plots divided into three subpopulations based on differences in genetic background: Mixed, temperate (TEM), and tropical or subtropical (TST). Of the 482 plots, 106 were from the mixed subpopulation, 162 were from the TEM subpopulation, and 214 were from the TST subpopulation.

The field trials were conducted at the research station of Xiao Tangshan National Precision Agriculture Research Center of China, Changping District of Beijing City $\left(115^{\circ} 50^{\prime} 17^{\prime \prime}-116^{\circ} 29^{\prime} 49^{\prime \prime} \mathrm{E}, \quad 40^{\circ} 20^{\prime} 18^{\prime \prime}-40^{\circ} 23^{\prime} 13^{\prime \prime} \mathrm{N}\right)$. The study area is in a warm temperate zone with a semihumid continental monsoon climate. The annual average temperature is $11.8{ }^{\circ} \mathrm{C}$, and the annual average precipitation is $550.3 \mathrm{~mm}$. Eight hundred plots were arranged across a field measuring approximately $30 \mathrm{~m}$ by $196 \mathrm{~m}$, with a spacing of $0.8 \mathrm{~m}$ between plots. Each plot consisted of three 2.4-m-long rows, each containing approximately 27 plants spaced $25 \mathrm{~cm}$ apart. All plots were planted by using a seeder on May 15, 2017.

\section{HTP platform and data acquisition}

The two cameras used in this study were mounted on an UAV HTP platform (DJI Spreading Wings S1000, SZ DJI Technology Co., Shenzhen, China). The first camera was a Sony digital RGB high-resolution camera (DSC-QX100, $5472 \times 3648$ pixels, Sony Electronics, Inc., Tokyo, Japan) with the ISO and shutter speed set at 160 and $1 / 2000$, respectively. The second camera was a Parrot Sequoia multispectral camera $(1280 \times 960$ pixels, MicaSense Inc., Seattle, USA) that combines four monochrome sensors (green: $550 \mathrm{~nm}$, red: $660 \mathrm{~nm}$, red-edge: $735 \mathrm{~nm}$, nearinfrared: $790 \mathrm{~nm}$ ) and can simultaneously capture four different band images with a $10 \mathrm{~nm}$ bandwidth (half maximum bandwidth) for the red-edge band and a $40 \mathrm{~nm}$ bandwidth for the green, red, and near-infrared bands. A sunshine sensor was used with the Sequoia sensors to minimize errors caused by variations in ambient light during acquisition (Fig. 1a).

Flight paths were designed by using the DJI ground station (SZ DJI Technology Co., Shenzhen, China) to ensure
$80 \%$ forward overlap and $75 \%$ side overlap, yielding six strips. To build the digital elevation model (DEM), the above-ground-level (AGL) parameter for the first flight was set to $40 \mathrm{~m}$, yielding a ground-sampling distance of $0.72 \mathrm{~cm}$. The AGL parameter for four other flights was set to $60 \mathrm{~m}$, yielding a ground-sampling distance of approximately $1.33 \mathrm{~cm}$. The radiometric calibration images were captured on the ground before and after each flight by using a calibrated reflectance panel (MicaSense Inc., Seattle, USA). Prior to the first flight, 16 ground control point (GCP) markers were arranged evenly over the experimental site and were measured by using a differential global positioning system (DGPS, South Surveying \& Mapping Instrument Co., Ltd., Guangzhou, China) with millimeter accuracy.

Images were acquired five times over the period spanning vegetative to reproductive growth (Fig. 1b). Table 1 details the flight conditions. The leaf collar method of Ritchie [44] was used for staging maize plant growth. Due to differences in genotype, heterogeneity appears in the growth and development at the plot scale. Therefore, the growth stage in Table 1 was determined by using the $50 \%$ majority rule.

\section{Plant sampling and measurements}

A total of 72 plots served as sampling plots for destructive biomass measurements and plant-height measurements. Because of the consumption caused by destructive sampling, another 72 plots were selected for the measurement on August 3, 2017 (Fig. 1c). Three plants were selected at random from the middle of the sampling plots to measure plant height and fresh biomass. Plant height was manually measured by using a telescopic leveling rod. The mean height of three plants was used as plant height at the plot scale for the ground truth. The three selected plants were then used for destructive biomass sampling. The fresh biomass was sealed in plastic bags and weighed on the same day. By calculating the actual number of plants per sampling plot, the mass was rescaled to $\mathrm{kg} / \mathrm{m}^{2}$. We visually determined the color of the positive leaves at the plot scale and recorded the results. From July 1 to 10,2017 , lodging occurred in some plots due to frequent strong winds and rainfall. A field investigation was done for 800 plots and the results for were recorded for root lodging, stem breaking, and stem lodging. Table 2 gives the dates for sampling and measurements.

\section{Image processing and data extraction}

Following UAV image acquisition, RGB images and multispectral images were processed by using two different software applications. For RGB images, we used Agisoft PhotoScan (version 1.3, Agisoft LLC, St. Petersburg, Russia) to generate orthomosaic and digital surface models 


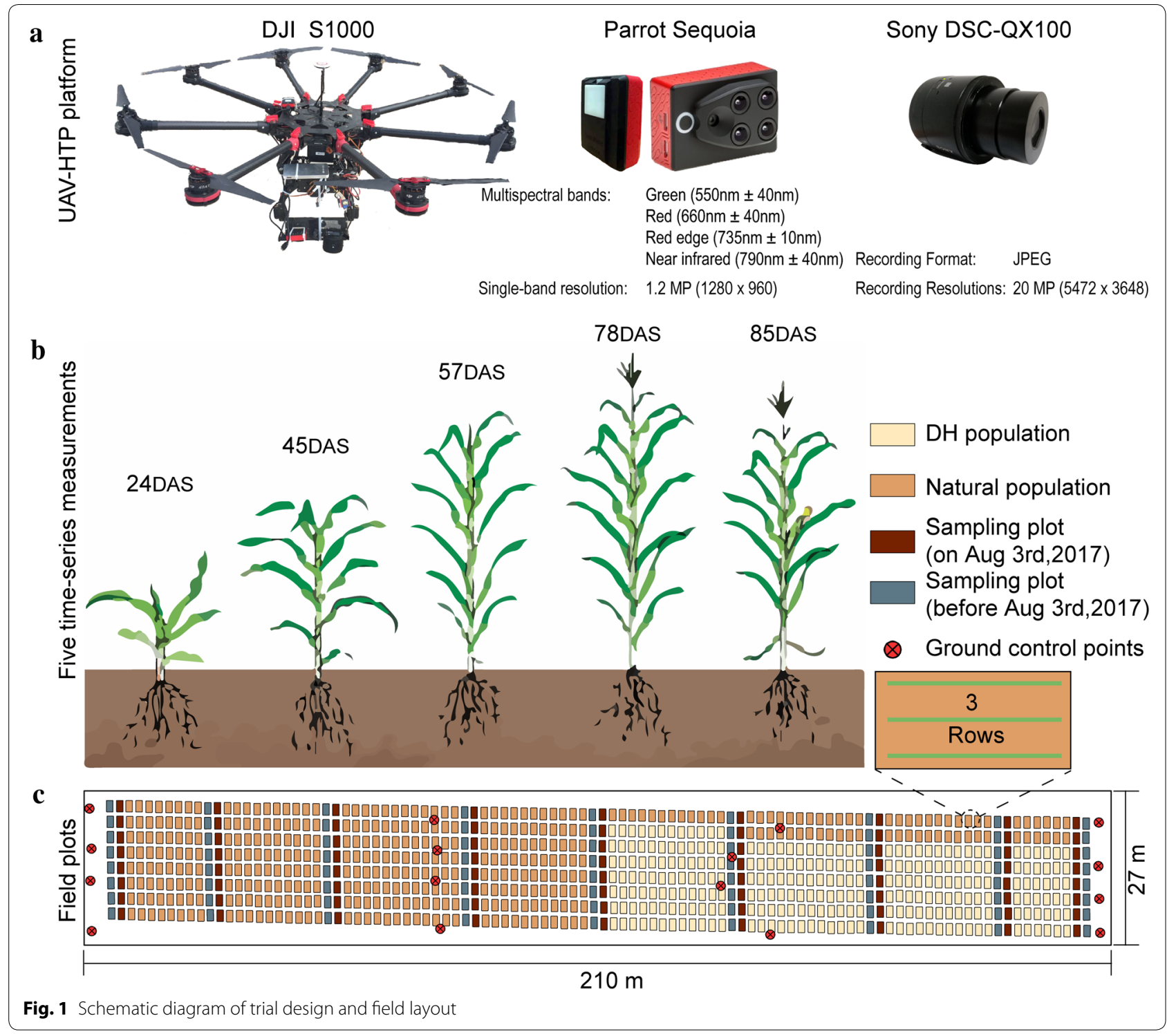

Table 1 Flight conditions for acquiring images over period spanning vegetative to reproductive growth

\begin{tabular}{lllll}
\hline Flight & Date & DAS $^{\mathbf{a}}$ & AGL & Growth stage \\
\hline 1 & $2017-06-08$ & 24 & 40 & V4 \\
2 & $2017-06-29$ & 45 & 60 & V10 \\
3 & $2017-07-11$ & 57 & 60 & V14 \\
4 & $2017-07-28$ & 78 & 60 & VT \\
5 & $2017-08-04$ & 85 & 60 & R1 \\
\hline
\end{tabular}

a Days after sowing

(DSMs) of each flight with the GCPs to optimize the camera position and ensure precise alignment [45]. A workflow (Han et al. 2018) was applied to create an area of interest for each plot by using the orthomosaics and to build the DEM by using the DSMs. Multispectral images were processed with Pix4D Mapper Pro software (version 4.0, PIX4D, Lausanne, Switzerland). Pix4D Mapper Pro has advantages in radiometric calibration and vegetation index calculation and offers some important processing steps similar to Agisoft Photoscan, such as aligning photos, importing GCPs and geographic references, building dense point clouds, and generating DSM and orthomosaics. Radiometric calibration was done by using the Pix4D Mapper Pro software with radiometric calibration images with known reflectance values provided by MicaSense. NDVI (or other vegetation indices) maps were then produced by using the index calculator.

Crop surface models (CSMs), which are widely used to extract crop height, were obtained by subtracting 
Table 2 Timing of plant sampling and measurement

\begin{tabular}{llll}
\hline Plant height & Fresh biomass & Lodging & Color \\
\hline $2017-06-29(45)$ & $2017-06-29(45)$ & - & $2017-06-30(46)$ \\
$2017-07-11(57)$ & $2017-07-11(57)$ & $2017-07-12(58)$ & - \\
$2017-07-29(79)$ & - & - & - \\
$2017-08-03(84)$ & $2017-08-03(84)$ & - & -
\end{tabular}

The date and days after sowing (in parentheses) are given for each task

the DEM from the DSMs. By using the areas of interest, a workflow [21] was applied to extract phenotypic data such as plant height and NDVI for each plot in the field from CSM and NDVI maps. Figure 2 illustrates the complete preprocessing chain.

After transforming a RGB image into HSL (i.e., hue, saturation and lightness) color space by using ENVI software (version 4.5, Esri Inc., Redlands, USA) and combining with field-sampled data, we used the hue value in HSL color space to cluster the population and classified the population into three leaf colors. Flowering was defined as a dichotomous variable that distinguishes whether flowering occurs at the fourth time point, as judged by visual observation of an orthomosaic. Average growth rate of plant height (AGRPH) is the increment in plant height per day between two adjacent time points [15]. Fresh biomass and BIOVP (a volume metric to estimate crop biomass within certain spatial ranges) were calculated following Han [21]. The identification of maize lodging was implemented following Han [29].

\section{Self-organizing map and hierarchical cluster analysis}

Selective breeding requires analysis of the relationships between multiple phenotypic traits and focuses on genotypes that are differentially expressed and co-expressed under the same environmental conditions. Differential expression can be accomplished using statistically significant difference test, while co-expressed genotypes require cluster analysis to examine the relationship between individuals or groups at the multiple-traits level. To explore co-expressed genotypes and identify underlying agronomic groups with similar phenotypic components, we performed two-step clustering to isolate 482 samples with nine dimensions that we standardized to values by using the mean and standard deviation. Twostep clustering was done for a pre-clustering by using the self-organizing map (SOM) method, which generates a simplified representation of the original data set and converts nonlinear statistical relationships between highdimensional data into simple geometric relationships between points on a two-dimensional map [46]. The pre-clusters were then subjected to agglomerative hierarchical clustering (AHC), which projects similar samples onto the same neuron. Finally, AHC analysis revealed the neighboring neurons of the topological map belonging to the same final cluster. A tree diagram was used to illustrate the arrangement of the clusters produced by AHC and to understand and identify clusters.

As shown in Fig. 3, the basic structure of the SOM network consists of an input layer and a competition layer. For mixed data in the input layer, an extra layer is created for each categorical variable, so difference distance measures for each layer. We used the Euclidean distance for the numerical variables and the Tanimato distance for the categorical variables, and then computed these distances for all weight vectors. For training, each neuron was associated with a weight vector (i.e., codebook) of the same dimensionality as the input vectors (i.e., phenotypic data), and the weight vector was updated at each iteration so that topological properties in the input layer were preserved [42].

We used the kohonen R package (version 3.0.8) [47] to perform the two-step clustering as follows [48]:

Step 0 Select the size (including size $\mathrm{X}$ and size $\mathrm{Y}$, i.e., the number of neurons), topology type (rectangular or hexagonal), and neighborhood function (Bubble or Gaussian).

Step 1 Each neuron is assigned a random codebook vector $\left(\vec{w}_{i}\right)$ with the same dimensionality $m$ as the input data $\left(\vec{x}_{n}\right)$.

Step 2 Select a data point at random from the training data and feed it into the SOM.

Step 3 Find the neuron whose codebook vector is most similar to the input data. This neuron is called the best matching unit (BMU). Similarity is calculated by using the Euclidean distance as numerical variable or the Tanimato distance as categorical variable.

Step 4 Move the BMU closer to the data point. The distance that the BMU moves is determined by the learning rate $\alpha$, which decreases after each iteration. Step 5 Adjust the codebook vector in the BMU's neighbors towards the chosen data point, depending on the neighborhood radius $r$ whose value decreases after each iteration.

Step 6 Update the learning rate $\alpha$ and neighborhood radius $r$, and repeat steps $2-5$ for $N$ iterations until the neuron positions stabilize.

Step 7 Cluster the stabilized codebook vectors by using AHC with Ward's minimum variance method linkage. The input data are separated into groups of similar properties, which are presented in different colors. Estimate the optimal number of clusters by using the NbClust R package (version 3.0) [49] with majority rule [50]. This provided 30 indices that 


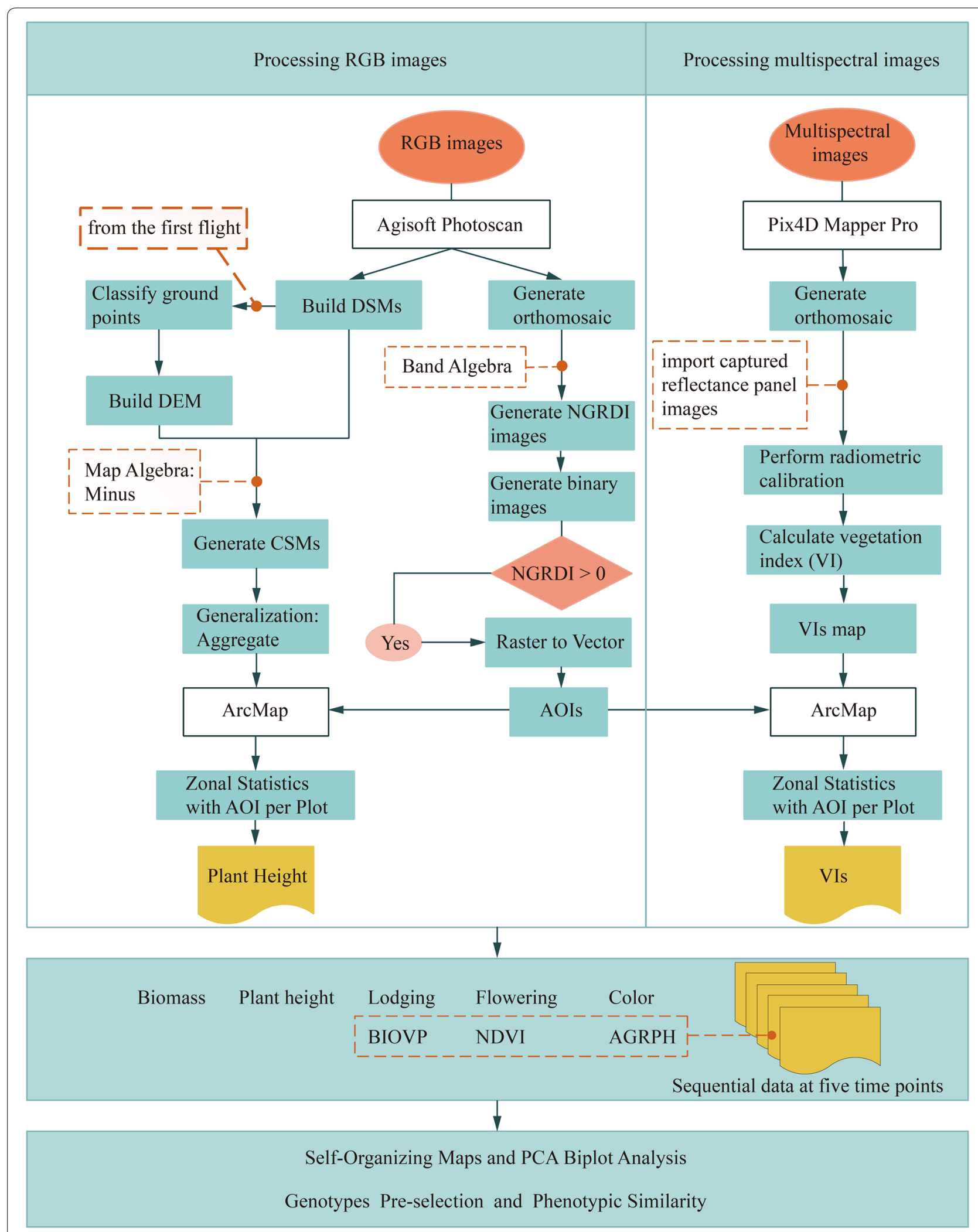

Fig. 2 Flowchart illustrating data-acquisition and analysis methodology 


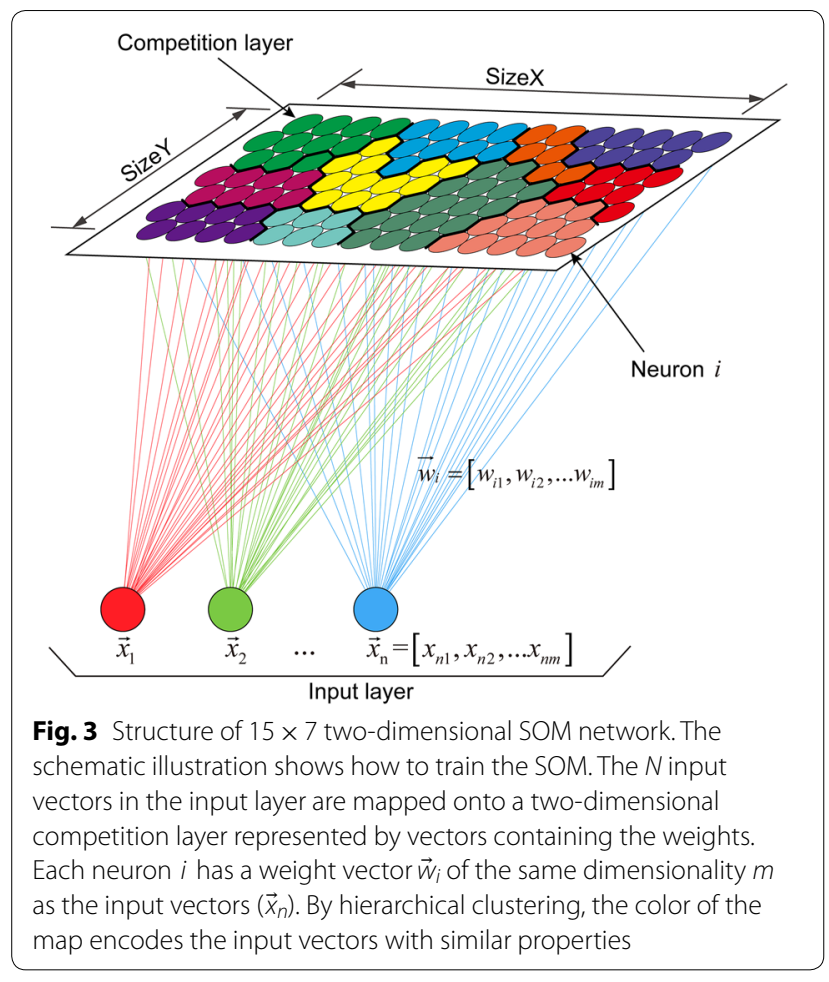

determine the number of clusters in a data set, but not all of the indices always work with all distance matrices, especially for a mix of numerical and categorical data. Therefore, only 20 applicable indices were used in the end (Additional file 1).

Finally, we ran SOM with the following parameters according to the guidelines for building a SOM reported by Das [51] and Wendel [52] : SOM size: $15 \times 7 ; 5000$ iterations; learning rate: 0.05 , hexagonal topology and Gaussian neighborhood function. We resolved the clusters derived from the SOM map into a set of clustering rules by using the rpart [53] (version 4.1-15) and rpart. plot [54] (version 3.0.7) R package and evaluated clustering quality. To facilitate interpretation of the clusters, we used the ComplexHeatmap R package (version 1.99.5) [55] to enhance the visualization of the clustering results by making a heat map with a dendrogram. The UpSetR R package (version 1.3.3) [56] used to visualize the set intersections was also used to identify clusters with typical phenotypic-component patterns.

Wilcoxon rank-sum test was used to compare each cluster mean with the total population (without clustering) mean, and observe whether a phenotypic component was overexpressed (above the total population mean) or underexpressed (below the total population mean) in different clusters.

\section{Analysis of principle components analysis bioplot}

Two-step clustering was followed by biplot analysis associated with principle components analysis (PCA). By using the FactoMineR [57] and factoextra [58] R packages, the biplot was analyzed with a new dataset based on two-step clustering to characterize the relationship between phenotypic components and to identify the leading components. Biplots based on simple bivariate scatter plots can show inter-unit distances and indicate clustering of units in addition to displaying variances in and correlations between the variables [59]. The new dataset was projected onto two dimensions to approximately preserve the distances between the samples. The points in the biplot approximate the row (sample) information and the vector approximates the column (i.e., phenotypic component) information. The distance between points reflects the difference between the corresponding samples. A greater distance between two points reflects a greater difference between the corresponding samples, and vice versa. The length of the arrows represents how well the phenotypic component explains the distribution of the data, whereas the angles between the arrows approximate their correlations. Therefore, when two vectors are approximately perpendicular, the correlation between the two variables is very weak, and they are essentially independent of each other. But if they are nearly parallel (antiparallel), the variables have a high positive (negative) correlation.

\section{Results}

Phenotypic components from high-throughput phenotyping images

The phenotypic components evaluated in this study included plant height, fresh biomass, flowering, lodging, leaf color, genetic background, NDVI, AGRPH and BIOVP. Except for genetic background, these phenotypic components were acquired by processing and analyzing digital or multispectral images from HTP. Manual grading based on field investigations led to extreme imbalance of the lodging samples, i.e., more than $80 \%$ was root lodging [29]. Therefore, we simply divided the population into two categories (i.e., lodging and non-lodging). Note that NDVI, BIOVP, and AGRPH were all time series data. For convenience, we converted the time series data into a numerical value by calculating the area under the polyline (Fig. 4). Phenotypic components were classified into three categories based on data types: numerical, dichotomous, and polytomous. Table 3 summarizes the phenotypic data evaluated in this study. 


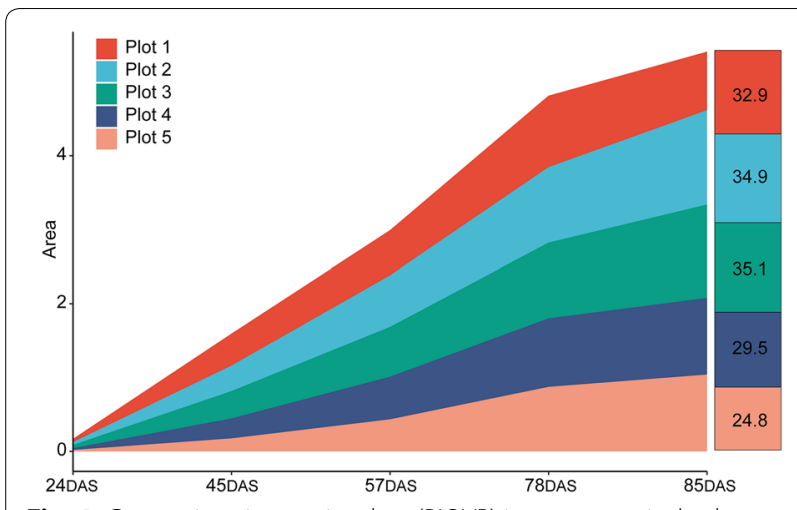

Fig. 4 Converting time series data (BIOVP) into a numerical value by calculating the area under the polyline. The first five plots were selected as examples. The number in the right column indicated the corresponding area after conversion

\section{Clustering genotypes based on phenotypic components}

There were five input data layers. The numerics layer consisted of five continuous numerical variables (i.e., dyAGRPH, dyNDVI, dyBIOVP, finBiomass and finPH, see Table 3). Figure 5 shows that the relative distance to the closest unit approximately stabilizes after 5000 iterations, which means that the algorithm has converged. A total of 105 neurons in the SOM were arranged in a grid of 15 rows by 7 columns, and 482 samples were unevenly distributed among these neurons (Fig. 6a). Samples with similar phenotypic-component patterns tended to be at nearby grid locations. The 91st neuron contained 35 samples, which was the largest number of samples for a single neuron. Seven neurons, called "dead" neurons, never won the competition for samples; these accounted for less than $7 \%$ (Fig. 6b). Figure 6c shows that, based on the majority rule, 11 of the 20 indices propose three as the optimal number of clusters. In other words, these phenotypic components contributed strongly to discrimination of genotypes into three clusters. Therefore, hierarchical

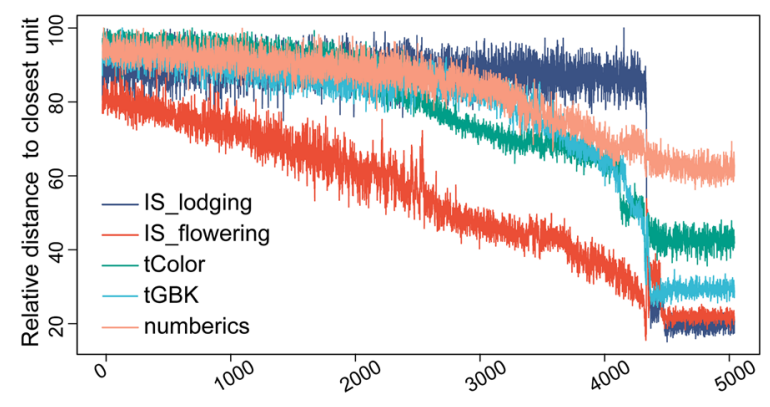

Fig. 5 Progress of SOM training iterations for 482 samples

agglomerative clustering of 105 codebooks resulted in the identification of three major clusters, with different genotype samples assigned to these clusters (Fig. 7). After clustering, genotypes with similar phenotypiccomponent characteristics were grouped in the same cluster. The next section discussed co-expressed genotypes and how to identify plant phenotypic similarity.

\section{Phenotypic map and similarity}

A phenotypic map was constructed by using SOM clustering visualization technology and dendrograms. This map provides important information regarding plant phenotypic similarity or dissimilarity and supports further evaluation of the phenotypic components [28]. By visualizing the weight vector on the map, we explored the patterns in which the samples and phenotypic components distributed across the three clusters. The weight vector was visualized as a "fan diagram" to indicate the weight of each phenotypic component in a neuron (see Figs. 8a-e). Closer inspection of these diagrams shows that (i) the weights of the numerical phenotypic components are relatively small in cluster 1 (Fig. 8a); (ii) the TST subpopulation dominates cluster 3 , accounting for more than 85\% (Fig. 8b); (iii) cluster 2 has almost no greenyellow samples (less than $5 \%$ of the samples), although

Table 3 Phenotypic components evaluated in this study

\begin{tabular}{lllll}
\hline Number & Variable & Sensor & Data type & Explanations \\
\hline 1 & finPH & RGB & Numerical & Plant height at fifth observation time point \\
2 & finBiomass & Both & Numerical & Fresh biomass at fifth observation time point \\
3 & IS_flowering & RGB & Dichotomous & Y means that the tassel has flowered, whereas N means that there is no tasseling \\
& IS_lodging & Both & Dichotomous tassel has not flowered & Y means lodging; N means no lodging \\
4 & tColor & RGB & Polytomous & Three colors: green, green-yellow, and dark green \\
5 & tGBK & - & Polytomous & Three genetic backgrounds: Mixed, temperate (TEM) and tropical-subtropical (TST) \\
6 & dyNDVI & Multispectral & Numerical & Converted from NDVI data across four time points \\
7 & dyBIOVP & RGB & Numerical & Converted from BIOVP data across five time points \\
9 & dyAGRPH & RGB & Numerical & Converted from AGRPH data across five time points \\
\hline
\end{tabular}




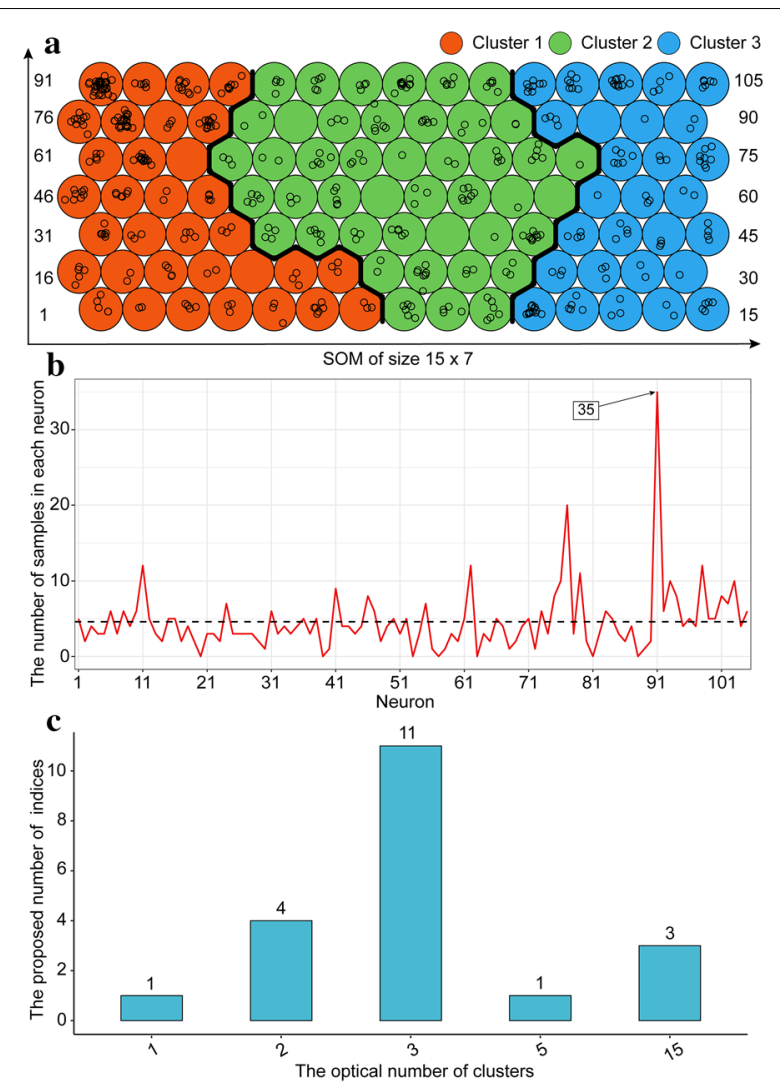

Fig. 6 Frequency distribution of samples in SOM grid and optical number of clusters. a SOM grid. The numbers on the left and right were the sequence numbers of 105 neurons. $\mathbf{b}$ Number of samples in each neuron. Seven neurons were dead neurons (i.e., neurons that never won the competition for samples). c Determining the optical number of clusters using the majority rule and 20 indices

they accounted for over $75 \%$ of the samples in cluster 3 (Fig. 8c); (iv) all samples containing lodging phenotypic components are in cluster 1 (Fig. 8d); and (v) none of the samples in cluster 3 are tasseling and flowering (Fig. 8e).

As shown in Fig. 9, the overall analysis of variance (ANOVA) gives a $p$ value less than 0.05 , so we further compared the differences in the mean phenotypic components between each cluster and that of all genotypes without clustering. When the Wilcoxon rank-sum test was significant, the numerical phenotypic components in cluster 1 were significantly low compared with all components (i.e., without clustering), and other numerical phenotypic components except $d y N D V I$ in Cluster 3 were significantly higher compared to all components. However, no significant difference appeared in the parameters finPH and $d y N D V I$ between cluster 2 and all components. Phenotypic components with similar characteristics were considered to be co-expressed by different genotypes. Together, the visualization of the results of the analysis provided important insights into the clusters and their co-expressed patterns based on two-step clustering (Table 4).

Turning now to phenotypic similarity, Fig. 7 provides an overview, via a dendrogram, of the measurement of the similarity between phenotypic components by using HAC with the spearman correlation distance. The top five principal components (PCs) explained $86.2 \%$ of the variation. A high Cos 2 (square cosine, squared coordinates) indicates that the phenotypic component is well represented in the principal component [58]. Therefore, ZfinBiomass (i.e., standardized fresh biomass) was more important to interpret PC 1, and IS_lodging was more important to interpret PC 3 (Fig. 10a). Figure 10b presents a biplot that simultaneously plots information on genotype samples and phenotypic components, further revealing the phenotypic similarity and the relationship between genotypes and phenotypic components. PC 1 explained $34.8 \%$ of the variation and PC 2 explained $19.0 \%$ of the variation. A high positive correlation existed between the numerical phenotypic components, which were negatively correlated with IS_lodging.Y. Lodging had a negative impact on crop biomass, plant height, NDVI, and so on. A significant positive correlation occurred between TST and IS_flowering.N and between TEM and IS_flowering. $N$, which we attributed to the fact that the experimental site was located in a temperate zone, so the TEM subpopulation was tasseling and flowering in the V1 stage, whereas the TST subpopulation required more accumulated temperature from vegetative overgrowth to reproductive growth. The correlation between greenyellow and IS_lodging. $Y$ was interesting because it seemed to indicate that the green-yellow leaf samples were more prone to lodging. Similarly, a significant positive correlation occurred between darkgreen and ZdyNDVI. Interestingly, this correlation was related to the canopy pigment content, which was remotely estimated based on the NDVI.

\section{Discussion}

\section{Phenotypic temporal profile}

Prior studies noted the importance of phenotypic temporal profiles, providing fresh insights into the dynamic changes in phenotypic traits $[15,60]$ and genetic differences at various stages of plant development [27, 61]. Although UAV-HTP allows researchers to efficiently and conveniently acquire multi-temporal phenotypic data by remote sensing, integrating time-series data with other types of phenotypic data, such as leaf color, to facilitate subsequent quantitative genetic analysis remains a difficult problem. In this study, the area under the polyline is highly correlated with other phenotypic components after conversion to a single value (Figs. 3 and 10). Although this conversion preserves the multi-temporal 


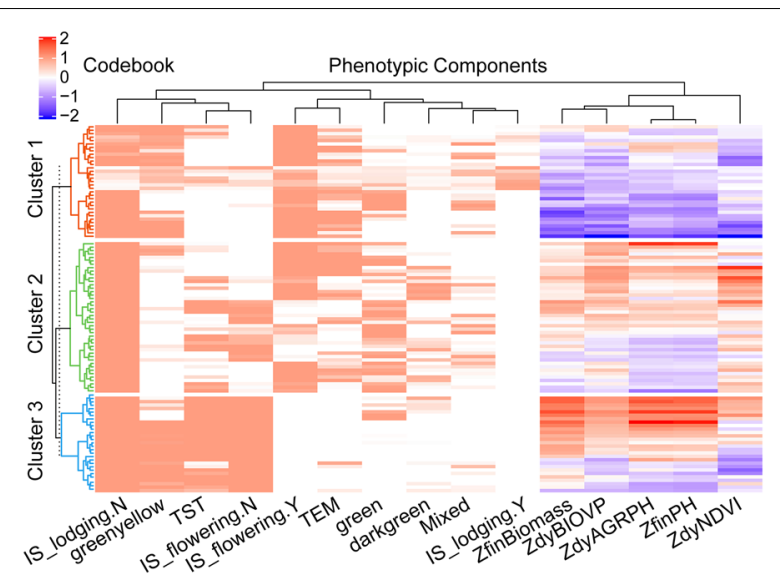

Fig. 7 Hierarchical agglomerative clustering for codebook. Clustering the codebooks resulted in the identification of three major clusters marked in three colors, to which different genotype samples had been assigned (left: clustering samples by rows). The dendrogram on the top showed the measure of phenotypic components similarity (clustering phenotypic components by columns). Converting categorical variables into dummy variables expanded to 15 the number of phenotypic components used for clustering. The initial letter "Z" indicated that this variable was standardized

information of phenotypic components to some extent, obvious deficiencies remain because, given equal areas, the shape of the polylines may differ. This leaves the way open for further improvements: one option would be to add some descriptive parameters such as inflection points and trends. The challenges of collecting phenotypic temporal profiles are further compounded by their observed values, which partially depend on environmental conditions and may change dramatically within a given day or between days of a given year [36].

\section{Phenotypic preselection}

The results of this study show that two-step clustering allows genotypes to be segregated according to different phenotypic component patterns. For example, cluster 3 exhibits a pattern that is a higher numerical phenotypic components, so when we preliminarily screen genotypes for higher biomass or higher plant height, which are both key breeding targets for crop improvement, we can narrow the range of candidate populations and select genotypes only in this cluster. Grain yield is also one of the most important breeding targets for breeders. Unfortunately, the grain-yield data for this trial were largely missing and unreliable due to waterlogging caused by heavy rainfall during the harvest period. These data were therefore replaced by the historical grain-yield data. In this way, we try to further explain the rationality of clustering results produced by our pre-selection framework.
Figure 11 shows the differences in grain weight per ear between the three genotype clusters. The most striking finding to emerge from this analysis is that cluster 3 has a lower grain weight per ear, despite previous analysis showing that it has higher phenotypic components. This finding seems to indicate that lower grain yields occur in genotype samples with higher plant height and higher biomass. Is this true? This result may be explained by the fact that the vast majority of genotypic samples $(85.2 \%)$ in cluster 3 are TST subpopulations planted in the temperate zone, where the grain yield of such TST populations was low due to the lack of sufficient accumulated temperature during the reproductive-growth stage. Grain yield is a polygenic trait controlled by several genes [62]; phenotypic components do not directly affect the yield but assist in the identification of the genotypes.

Phenotypes of a plant are the expression in observable traits of potential genes comprising the genotype and are determined by its genetic composition, the environment in which it grows, and the interactions between genotype and environment [63,64]. It is thus possible to measure phenotypic relationships between different genotypes based on available traits [28]. Some phenotypic data extracted from remote sensing images, such as BIOVP and NDVI, clearly differ from the agronomic definition, or "trait." To distinguish between them, we refer herein to these phenotypic data as phenotypic components. The analysis of phenotypic similarity provides a new inspiration that removes some highly relevant phenotypic components and thereby reduces data redundancy. In other words, the workload can be reduced by making a preliminarily selection of representative phenotypic components as the research objects in the trial design.

\section{Limitations and Implications of Study}

According to the literature, SOM does not really cluster but instead produces a reduced representation of the original data set [46]. Because SOM lacks hierarchical structure, it is impossible to detect higher-order relationships between clusters. In this study, we demonstrate a two-step clustering method that combines hierarchical clustering and self-organizing maps to cluster, analyze, and visualize the mixed continuous and categorical data, providing a very efficient tool for exploratory analysis of genotype co-expression and phenotypic similarity. One problem with self-organizing maps is the occurrence of dead neurons, whose randomly initialized weight vectors farther from any data point prevent them from ever being chosen as BMU, which degrades the learning efficiency. Therefore, if the percentage of dead neurons is large, caution must be applied and the grid size of SOM should be readjusted. After resolving the clusters derived from the SOM map reasonably to a set of clustering rules, 

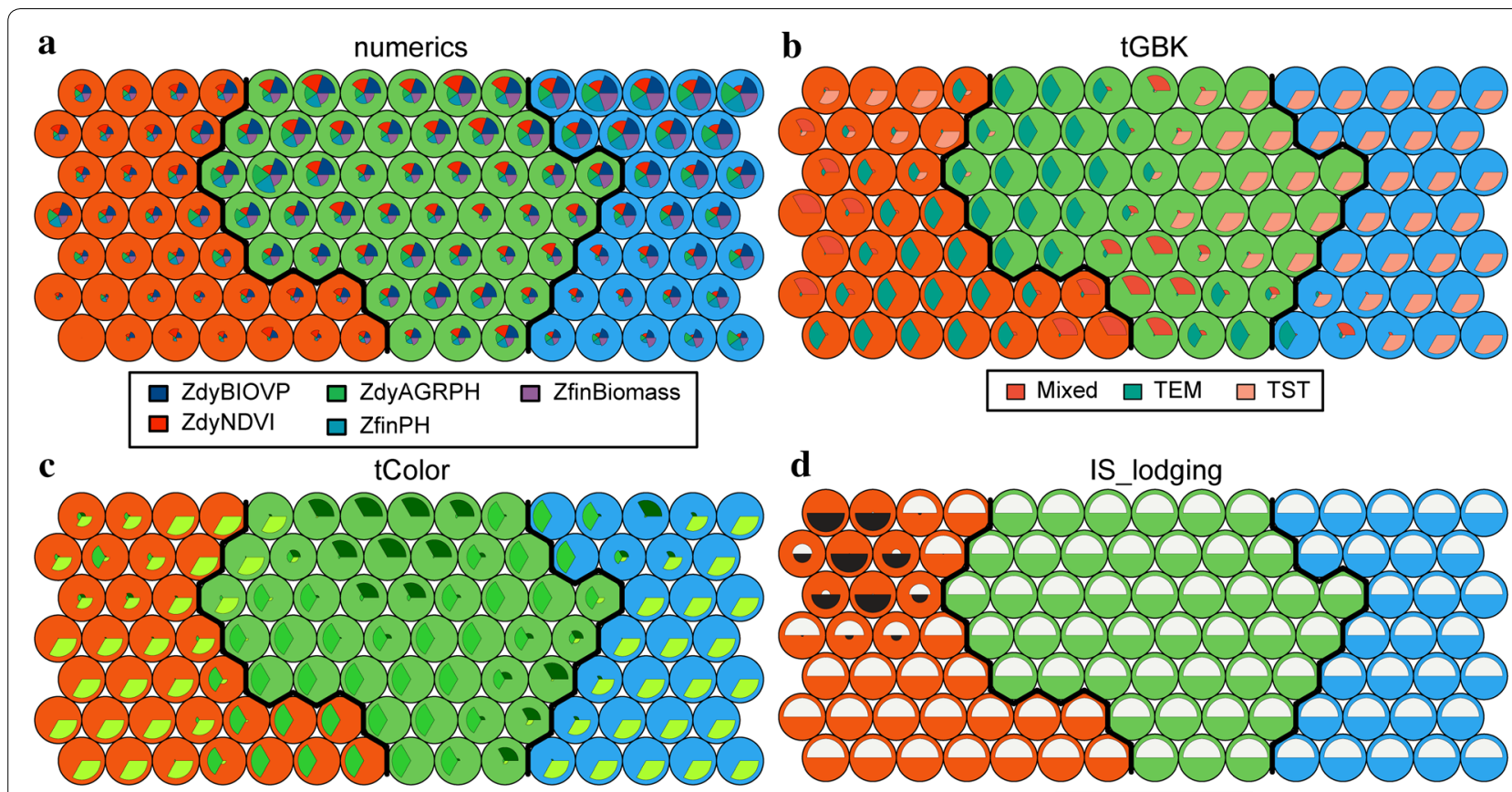

$\square$ darkgreen $\square$ green $\square$ greenyellow

e

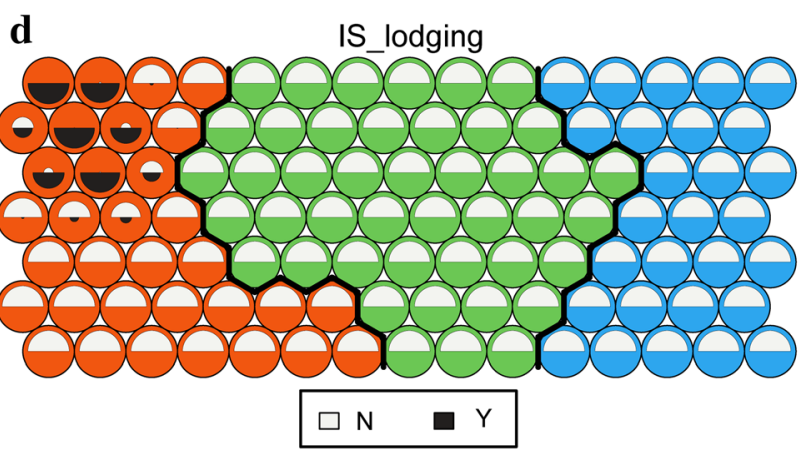

f
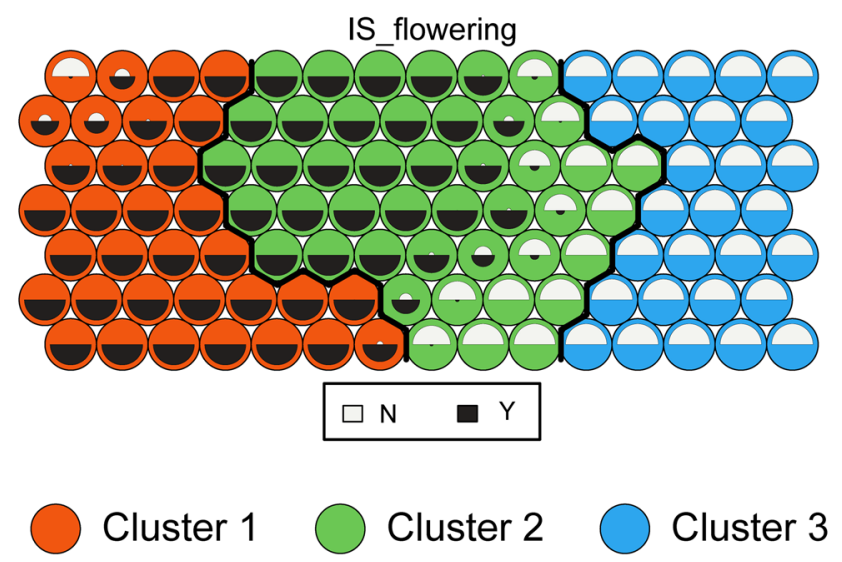

IS_flowering. $\stackrel{0}{N}$
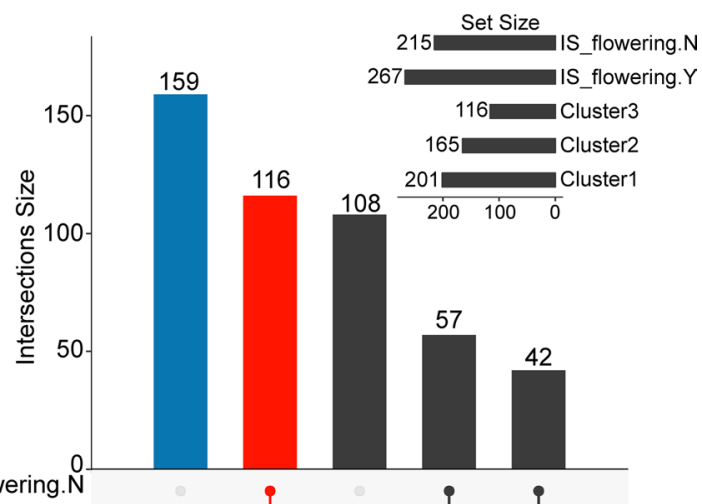

IS_flowering.Y

Cluster3

Cluster2

Cluster1

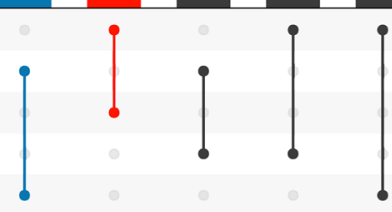

Fig. 8 Exploring the distribution patterns of sample and phenotypic components in clusters. a-e The weight vector was visualized as a fan diagram to indicate the weight of each phenotypic component in a neuron. f Taking IS_flowering as an example, the phenotypic component patterns within clusters were identified to reveal the effect of co-expressed genotypes. The intersection of cluster 3 and the IS_flowering.N set gave 116 samples, which indicated that none of the samples in Cluster 3 were tasseling and flowering, accounting for $100 \%$ of the samples. This was shown with a striking color to distinguish between unrecognized patterns. The initial letter "Z" indicated that this variable was standardized

approximately $95.2 \%$ of the samples can be accurately predicted by using a decision tree. However, the clustering boundaries are not $100 \%$ sharp, and some ambiguity remains regarding where on the map a specific neuron (sample) migrates (Fig. 12).

The challenge for plant breeders is to identify and select the plants with the target phenotype controlled by the corresponding genotypes, rather than plants with the target phenotype controlled by environmental impact [65]. For field breeding trials, an effective use of phenotypic data remains challenging, because the environment changes daily throughout the data-collection period [66]. To minimize the impact of the environment on this study, comparatively ideal environmental 


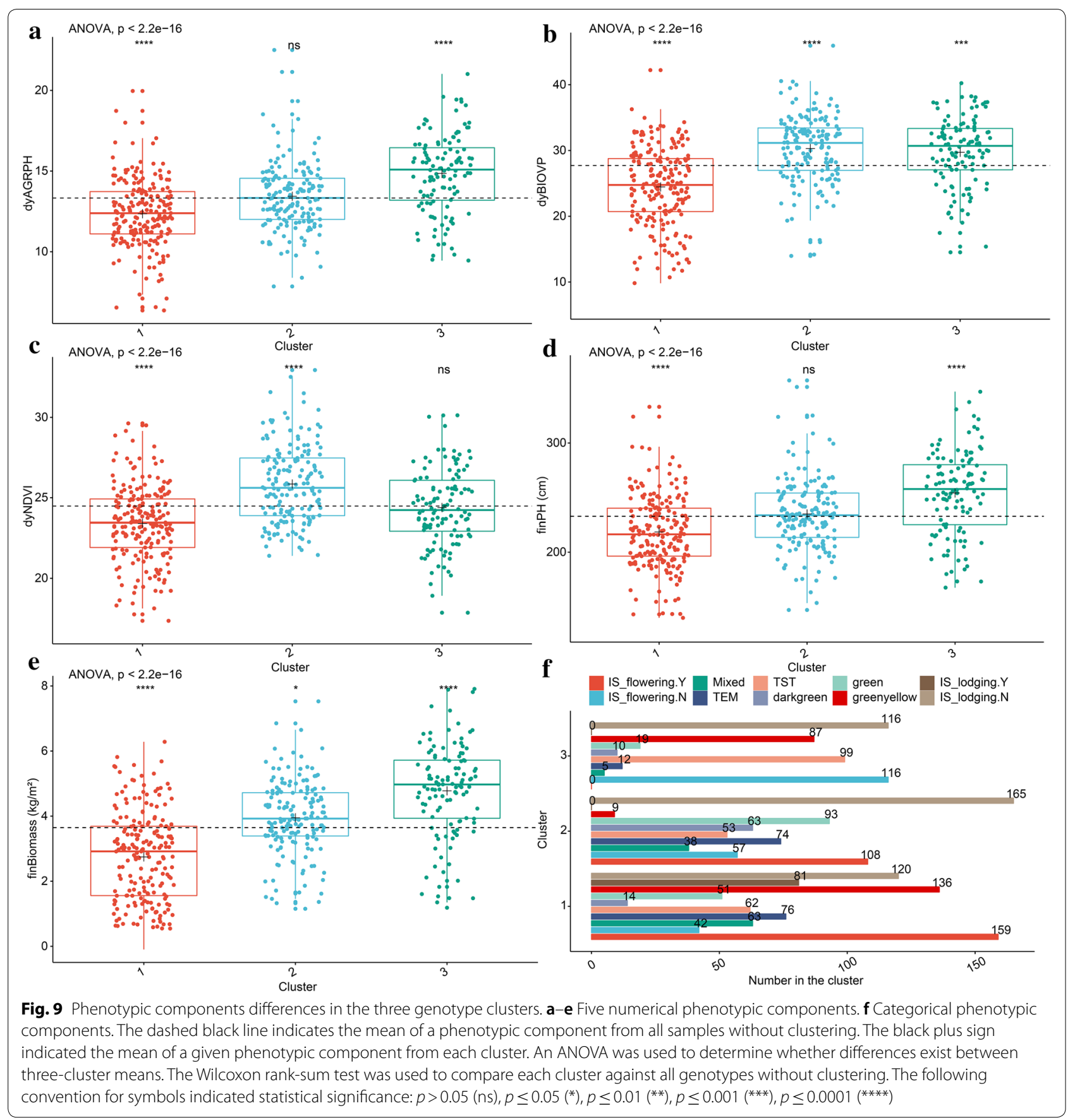

conditions were attained by doing single-factor experiments and using uniform field-management practices. Even so, the explanation for these results may be the lack of adequate verification, because these phenotypic components were acquired and analyzed only across a single growing season. Further research should be undertaken to investigate the population and provide new multi-year insights into identifying genotypes and their co-expressed patterns and selecting phenotypic similarity.

The capacity of UAV-based HTP to collect and analyze from a few to thousands of breeding plots allows breeders to effortlessly obtain a large number of phenotypic data, and then to efficiently quantify genotypic differences in crop-yield potential, stress resistance, and quality. In this study, the lack of phenotypic data 
Table 4 Summary of clusters and their co-expressed patterns based on two-step clustering

\begin{tabular}{llll}
\hline Cluster & Neuron size & Sample size & Description \\
\hline 1 & 33 & 201 & $\begin{array}{l}\text { Lower numerical phenotypic components, e.g., less fresh biomass and lower average } \\
\text { growth rate of plant height } \\
\text { Green or dark green leaves, almost no green-yellow leaves } \\
\text { Larger NDVI } \\
\text { Higher numerical phenotypic components, e.g., more fresh biomass and plant height } \\
\text { Longer vegetative growth stage }\end{array}$ \\
\hline
\end{tabular}

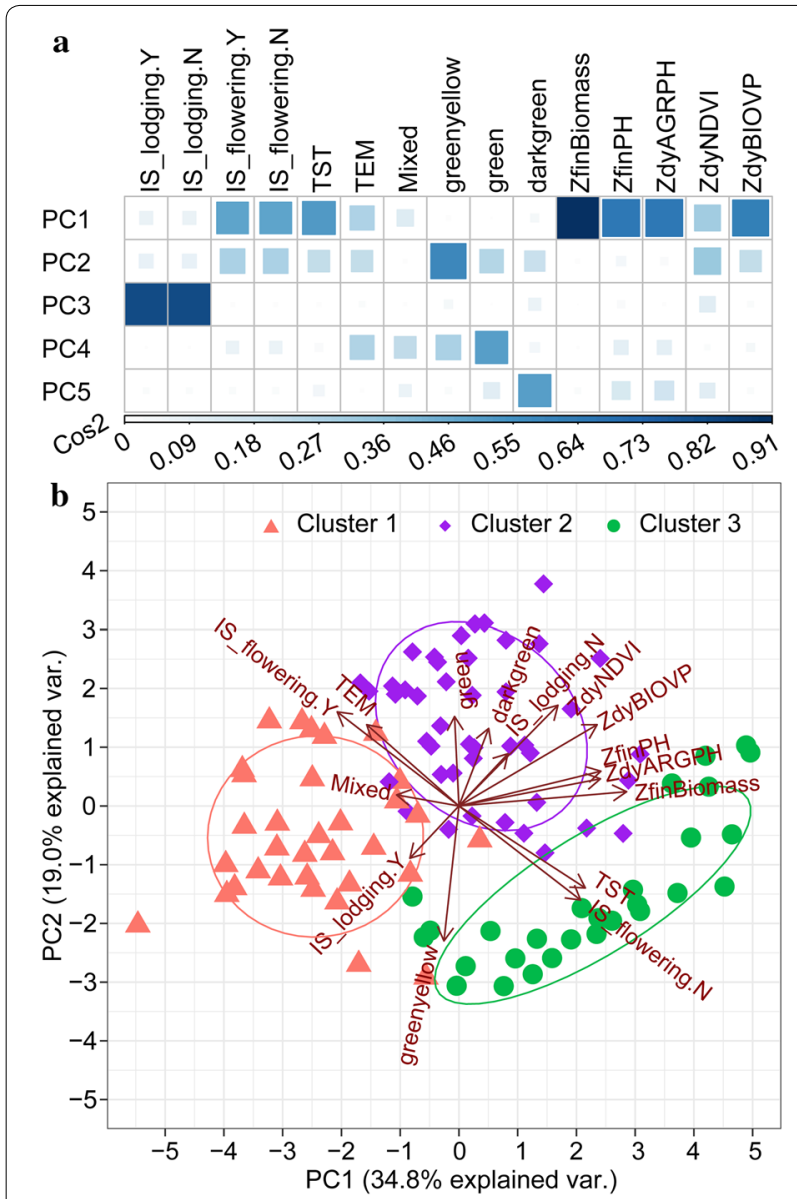

Fig. 10 PCA biplot showing phenotypic similarity and relationship between genotypes and phenotypic components. a Visualization of quality of representation of variables in top five dimensions. $\cos 2$ is the quality of representation of the variables in the principal component maps. b Biplot analysis for phenotypic similarity. Correlated phenotypic components and genotype samples were located in the same quadrant. The cosine of the angle between vectors indicated correlation between phenotypic components. Highly correlated phenotypic components pointed in roughly the same direction. Nearby points in the biplot represented samples with similar patterns; these were colored according to clustering. The initial letter "Z" indicated that this variable is standardized

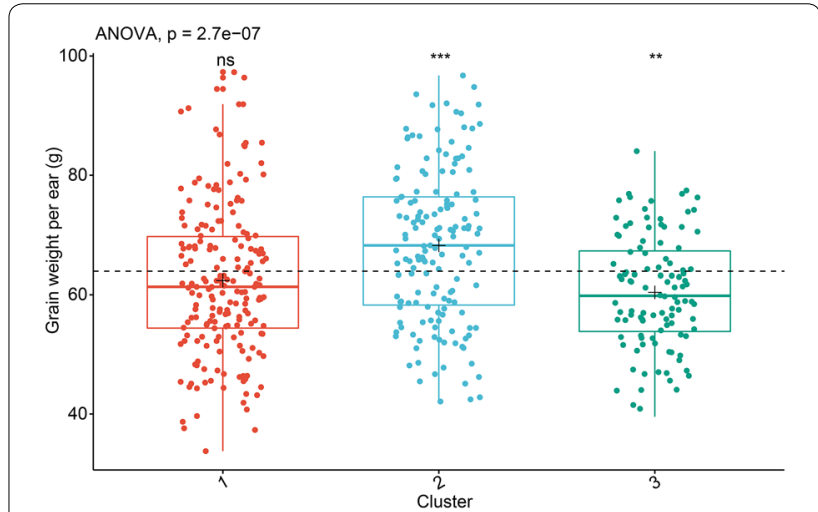

Fig. 11 Differences in grain weight per ear between the three genotype clusters. The dashed black line indicated the mean of a phenotypic component from all samples without clustering. The black plus sign indicated the mean of a given phenotypic component from each cluster. An ANOVA was used to determine whether differences exist between three-cluster means. The Wilcoxon rank-sum test was used to compare each cluster against all genotypes without clustering. The following convention for symbols indicated statistical significance: $p>0.05$ (ns); $p \leq 0.01\left(^{* *}\right) ; p \leq 0.001$ $(* * *)$. The total sample size remains 453 , excluding missing values

at the late ripening stage prevented the formation of a phenotypic data chain throughout growing season. A hyperspectral sensor is a powerful tool for detecting biological and abiotic stresses, and more crop resistance information can be obtained when it is loaded on the UAV platform. The platform will also be improved to increase payload capacity so that it may be equipped with UAV laser scanning for easy access to plant type and ear-high traits. The phenotypic map and similarity will also more comprehensive when more data from UAV laser scanning and hyperspectral sensor are available. Rich phenotypic data definitely assist breeders with identifying and selecting the best candidate genotypes. 


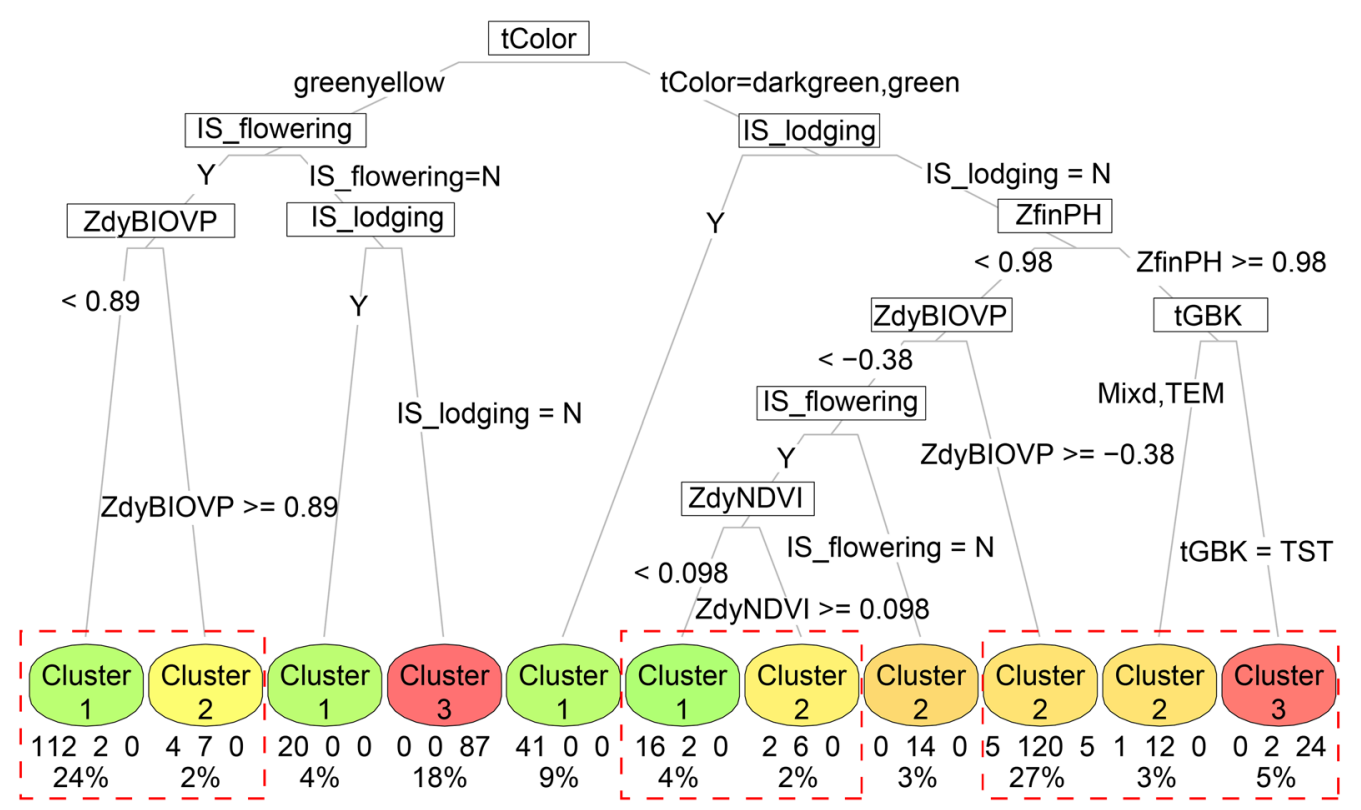

Fig. 12 Clustering resolved into decision tree. Each node displayed the predicted class, the number, and the percent of samples. The red-dashed-line rectangle showed a situation where clustering boundaries were blurred

\section{Conclusions}

In this study, we collected for a maize breeding program a short time series of remote-sensing images, including digital and multispectral images, by using an UAV-based HTP platform. The images were used to acquire nine phenotypic components. Here, we propose a framework for pre-screening genotypes and phenotypes based on HTP phenotypic components. The core procedure of this framework can be summarized as follows: we use two-step clustering to identify co-expressed patterns, and then pre-select genotypes. We then use correlation analysis to analyze phenotypic similarity, and then preselect phenotypic components. This framework gives breeders additional information to quickly identify and select plants that have genotypes that confer desirable phenotypic components from thousands of field plots. The present study also demonstrates that remote sensing is a powerful tool that provides an opportunity to acquire abundant phenotypic components. By using these rich phenotypic components, breeders should be able to more effectively identify and select superior genotypes.

\section{Additional file}

Additional file 1. Twenty indices for determining the best number of clusters.

\section{Abbreviations}

AGRPH: average growth rate of plant height; AHC: agglomerative hierarchical clustering; ANOVA: analysis of variance; AOI: area of interest; BIOVP: a volume metric used to estimate crop biomass within a plot; Cos2: square cosine and squared coordinates; CSM: crop surface model; DAS: days after sowing; DSM: digital surface model; DEM: digital elevation model; DSMs: digital surface models; HTP: high-throughput phenotyping; NGRDI: normalized green-red difference index; NDVI: normalized difference vegetation index; PCA: principle components analysis; SOM: self-organizing map; UAV: unmanned aerial vehicle; Vls: vegetation indices.

\section{Acknowledgements}

We thank the Maize Research Center department of the Beijing Academy of Agriculture and Forestry Sciences for preparing the seed and planting for the trial, and Dr. Yanxin Zhao, Dr. Xiaging Wang, and Mr. Ruyang Zhang for designing the experiments and helping to collect the field data. We are also grateful to the anonymous reviewers for their valuable comments and recommendations.

\section{Authors' contributions}

LH drafted and revised the manuscript. GY and CZ proposed the conceptualization of this study and reviewed the manuscript. HD edited the manuscript. $\mathrm{HY}$ and $\mathrm{LH}$ performed field experiments. BX collected image data. $\mathrm{LH}, \mathrm{ZL}$ and $\mathrm{XY}$ analyzed and interpreted the results. $\mathrm{HL}$ and $\mathrm{HL}$ assisted in developing analysis methods and in revising the manuscript. All authors read and approved the final manuscript.

\section{Funding}

This study was supported by the National Key Research and Development Program of China (2017YFE0122500), the Natural Science Foundation of China (41771469), the Beijing Natural Science Foundation (6182011), and the Special Funds for Technology innovation capacity building sponsored by the Beijing Academy of Agriculture and Forestry Sciences (KJCX20170423).

\section{Availability of data and materials}

The datasets analysed during the current study are available from the corresponding author on reasonable request. 
Ethics approval and consent to participate

Not applicable.

\section{Consent for publication}

Not applicable.

\section{Competing interests}

The authors declare that they have no competing interests.

\begin{abstract}
Author details
${ }^{1}$ Key Laboratory of Quantitative Remote Sensing in Agriculture of Ministry of Agriculture, Beijing Research Center for Information Technology in Agriculture, Beijing 100097, China. ${ }^{2}$ College of Architecture and Geomatics Engineering, Shanxi Datong University, Datong 037003, China. ${ }^{3}$ National Engineering Research Center for Information Technology in Agriculture, Beijing 100097, China. ${ }^{4}$ College of Geoscience and Surveying Engineering, China University of Mining and Technology (Beijing), Beijing 100083, China.
\end{abstract}

Received: 29 March 2019 Accepted: 22 May 2019

Published online: 28 May 2019

\section{References}

1. Furbank RT, Tester M. Phenomics - technologies to relieve the phenotyping bottleneck. Trends Plant Sci. 2011;16(12):635-44.

2. Ninomiya S, Baret F, Cheng Z-MM. Plant phenomics: emerging transdisciplinary science. Plant Phenom. 2019;2019:1-3. https://doi.org/10.34133 /2019/2765120.

3. The UN Food and Agriculture Organisation (FAO). How to Feed the World in 2050. 2009: Rome. p. 35

4. Yang G, Liu J, Zhao C, Li Z, Huang Y, Yu H, Xu B, Yang X, Zhu D, Zhang X, Zhang R, Feng H, Zhao X, Li Z, Li H, Yang H. Unmanned aerial vehicle remote sensing for field-based crop phenotyping: current status and perspectives. Front Plant Sci. 2017:8:1111.

5. Cobb JN, DeClerck G, Greenberg A, Clark R, McCouch S. Next-generation phenotyping: requirements and strategies for enhancing our understanding of genotype-phenotype relationships and its relevance to crop improvement. Theor Appl Genet. 2013;126(4):867-87.

6. Araus JL, Cairns JE. Field high-throughput phenotyping: the new crop breeding frontier. Trends Plant Sci. 2014;19:52-61.

7. Campbell ZC, Acosta-Gamboa LM, Nepal N, Lorence A. Engineering plants for tomorrow: how high-throughput phenotyping is contributing to the development of better crops. Phytochem Rev. 2018;17:1329-43.

8. Fernandez MGS, Bao Y, Tang L, Schnable PS. A high-throughput, fieldbased phenotyping technology for tall biomass crops. Plant Physiol. 2017;174(4):2008-22.

9. Araus JL, Kefauver SC, Zaman-Allah M, Olsen MS, Cairns JE. Translating high-throughput phenotyping into genetic gain. Trends Plant Sci. 2018;23(5):451-66.

10. Crain JL, Wei Y, Barker J, Thompson SM, Alderman PD, Reynolds M, Zhang NQ, Poland J. Development and deployment of a portable field phenotyping platform. Crop Sci. 2016;56(3):965-75.

11. Bai G, Ge YF, Hussain W, Baenziger PS, Graef G. A multi-sensor system for high throughput field phenotyping in soybean and wheat breeding. Comput Electron Agric. 2016;128:181-92.

12. White JW, Andrade-Sanchez P, Gore MA, Bronson KF, Coffelt TA, Conley MM, Feldmann KA, French AN, Heun JT, Hunsaker DJ, Jenks MA, Kimball BA, Roth RL, Strand RJ, Thorp KR, Wall GW, Wang G. Field-based phenomics for plant genetics research. Field Crops Res. 2012;133:101-12.

13. Ludovisi R, Tauro F, Salvati R, Khoury S, Mugnozza Scarascia G, Harfouche A. UAV-based thermal imaging for high-throughput field phenotyping of black poplar response to drought. Front Plant Sci. 2017:8:1681-81.

14. Maimaitijiang M, Ghulam A, Sidike P, Hartling S, Maimaitiyiming M, Peterson K, Shavers E, Fishman J, Peterson J, Kadam S, Burken J, Fritschi F. Unmanned Aerial System (UAS)-based phenotyping of soybean using multi-sensor data fusion and extreme learning machine. ISPRS J Photogramm Remote Sens. 2017;134:43-58.

15. Han L, Yang G, Yang H, Xu B, Li Z, Yang X. Clustering field-based maize phenotyping of plant-height growth and canopy spectral dynamics using a UAV remote-sensing approach. Front Plant Sci. 2018;9:1638.
16. Haghighattalab A, Perez LG, Mondal S, Singh D, Schinstock D, Rutkoski J, Ortiz-Monasterio I, Singh RP, Goodin D, Poland J. Application of unmanned aerial systems for high throughput phenotyping of large wheat breeding nurseries. Plant Methods. 2016;12:15.

17. Deng L, Mao Z, Li X, Hu Z, Duan F, Yan Y. UAV-based multispectral remote sensing for precision agriculture: a comparison between different cameras. ISPRS J Photogramm Remote Sens. 2018;146:124-36.

18. Khan Z, Rahimi-Eichi V, Haefele S, Garnett T, Miklavcic SJ. Estimation of vegetation indices for high-throughput phenotyping of wheat using aerial imaging. Plant Methods. 2018;14(1):20.

19. Aasen H, Honkavaara E, Lucieer A, Zarco-Tejada PJ. Quantitative remote sensing at ultra-high resolution with UAV spectroscopy: a review of sensor technology, measurement procedures, and data correction workflows. Remote Sens. 2018;10(7):1091.

20. Ampatzidis Y, Partel V. UAV-based high throughput phenotyping in citrus utilizing multispectral imaging and artificial intelligence. Remote Sens. 2019;11(4):410.

21. Han L, Yang G, Dai H, Xu B, Yang H, Feng H, Li Z, Yang X. Modeling maize above-ground biomass based on machine learning approaches using UAV remote-sensing data. Plant Methods. 2019;15(1):10.

22. Crain J, Reynolds M, Poland J. Utilizing high-throughput phenotypic data for improved phenotypic selection of stress-adaptive traits in wheat. Crop Sci. 2017;57(2):648-59.

23. Duan T, Chapman SC, Guo Y, Zheng B. Dynamic monitoring of NDVI in wheat agronomy and breeding trials using an unmanned aerial vehicle. Field Crops Res. 2017;210:71-80

24. Zhang XH, Huang CL, Wu D, Qiao F, Li WQ, Duan LF, Wang K, Xiao YJ, Chen GX, Liu Q, Xiong LZ, Yang WN, Yan JB. High-throughput phenotyping and QTL mapping reveals the genetic architecture of maize plant growth. Plant Physiol. 2017;173(3):1554-64.

25. Watanabe K, Guo W, Arai K, Takanashi H, Kajiya-Kanegae H, Kobayashi M, Yano K, Tokunaga T, Fujiwara T, Tsutsumi N, Iwata H. High-throughput phenotyping of sorghum plant height using an unmanned aerial vehicle and its application to genomic prediction modeling. Front Plant Sci. 2017:8:11.

26. Hu P, Chapman SC, Wang X, Potgieter A, Duan T, Jordan D, Guo Y, Zheng B. Estimation of plant height using a high throughput phenotyping platform based on unmanned aerial vehicle and self-calibration: example for sorghum breeding. Eur J Agron. 2018;95:24-32.

27. Pugh NA, Horne DW, Murray SC, Carvalho G, Malambo L, Jung J, Chang A, Maeda M, Popescu S, Chu T, Starek MJ, Brewer MJ, Richardson G, Rooney WL. Temporal estimates of crop growth in sorghum and maize breeding enabled by unmanned aerial systems. Plant Phenome J. 2018;1(1):170006.

28. Chen D, Neumann K, Friedel S, Kilian B, Chen M, Altmann T, Klukas C. Dissecting the phenotypic components of crop plant growth and drought responses based on high-throughput image analysis. Plant Cell. 2014;26(12):4636-55.

29. Han L, Yang G, Feng H, Zhou C, Yang H, Xu B, Li Z, Yang X. Quantitative identification of maize lodging-causing feature factors using unmanned aerial vehicle images and a nomogram computation. Remote Sens. 2018;10(10):1528

30. Liu T, Li R, Zhong X, Jiang M, Jin X, Zhou P, Liu S, Sun C, Guo W. Estimates of rice lodging using indices derived from UAV visible and thermal infrared images. Agric For Meteorol. 2018;252:144-54.

31. Friedman JM, Hunt ER, Mutters RG. Assessment of leaf color chart observations for estimating maize chlorophyll content by analysis of digital photographs. Agron J. 2016;108(2):822-9.

32. Vergara-Diaz O, Zaman-Allah MA, Masuka B, Hornero A, Zarco-Tejada P, Prasanna BM, Cairns JE, Araus JL. A novel remote sensing approach for prediction of maize yield under different conditions of nitrogen fertilization. Front Plant Sci. 2016;7:666.

33. Naito H, Ogawa S, Valencia MO, Mohri H, Urano Y, Hosoi F, Shimizu Y, Chavez AL, Ishitani M, Selvaraj MG, Omasa K. Estimating rice yield related traits and quantitative trait loci analysis under different nitrogen treatments using a simple tower-based field phenotyping system with modified single-lens reflex cameras. ISPRS J Photogramm Remote Sens. 2017;125:50-62.

34. Yu N, Li L, Schmitz N, Tian LF, Greenberg JA, Diers BW. Development of methods to improve soybean yield estimation and predict plant maturity with an unmanned aerial vehicle based platform. Remote Sens Environ. 2016:187:91-101. 
35. Wang X, Singh D, Marla S, Morris G, Poland J. Field-based high-throughput phenotyping of plant height in sorghum using different sensing technologies. Plant Methods. 2018;14(1):53.

36. Pauli D, Andrade-Sanchez P, Carmo-Silva AE, Gazave E, French AN, Heun J, Hunsaker DJ, Lipka AE, Setter TL, Strand RJ, Thorp KR, Wang S, White JW Gore MA. Field-based high-throughput plant phenotyping reveals the temporal patterns of quantitative trait loci associated with stress-responsive traits in cotton. Genes Genomes Genetics. 2016;6(4):865-79.

37. Tagliotti ME, Deperi SI, Bedogni MC, Zhang R, Manrique Carpintero NC, Coombs J, Douches D, Huarte MA. Use of easy measurable phenotypic traits as a complementary approach to evaluate the population structure and diversity in a high heterozygous panel of tetraploid clones and cultivars. BMC Genet. 2018;19(1):8.

38. Oyelade J, Isewon I, Oladipupo F, Aromolaran O, Uwoghiren E, Ameh F, Achas M, Adebiyi E, Clustering algorithms: their application to gene expression data. Bioinform Biol Insights 2016. 10: p. BBI.S38316.

39. Higuera C, Gardiner KJ, Cios KJ. Self-organizing feature maps identify proteins critical to learning in a mouse model of down syndrome. PLoS ONE. 2015;10(6):e0129126.

40. Kohonen T. Automatic formation of topological maps in a self-organizing system. In Proceedings of the Scandinavian Conference on Image Analysis. 1981.

41. Kohonen T. Exploration of very large databases by self-organizing maps. In Proceedings of International Conference on Neural Networks (ICNN'97). 1997.

42. Augustijn E-W, Zurita-Milla R. Self-organizing maps as an approach to exploring spatiotemporal diffusion patterns. Int J Health Geogr. 2013;12:60-61.

43. Bro R, Smilde AK. Principal component analysis. Anal Methods. 2014;6(9):2812-31.

44. Ritchie S W, Hanway J J, and Benson G O, How a corn plant develops, in Special Report \#48. 1993, lowa State University of Science and Technology Cooperative Extension Service: Ames, IA, USA.

45. Agisoft. Orthophoto \& DEM Generation (with GCPs). 2018 [cited 201819 October,2018]. http://www.agisoft.com/support/tutorials/beginner-level /.

46. Herrero J, Dopazo J. Combining hierarchical clustering and self-organizing maps for exploratory analysis of gene expression patterns. J Proteome Res. 2002;1 (5):467-70

47. Wehrens R, Buydens LMC. Self- and super-organizing maps in R: The kohonen Package. J Stat Softw. 2007;21(5):19.

48. Algobeans. Self-organizing maps tutorial. n.d. [cited 2019 January, 12] https://algobeans.com/2017/11/02/self-organizing-map/.

49. Charrad M, Ghazzali N, Boiteau V, Niknafs A. NbClust: an R package for determining the relevant number of clusters in a data set. J Stat Softw. 2014;61(6):36.

50. Yang Y. Ensemble learning. In: Yang Y, editor. temporal data mining via unsupervised ensemble learning. Amsterdam: Elsevier; 2017. p. 35-56.

51. Das G, Chattopadhyay M, Gupta S. A comparison of self-organising maps and principal components analysis. Int J Market Res. 2016;58(6):815-34
52. Wendel J, Buttenfield BP. Formalizing guidelines for building meaningful self-organizing maps. In GIScience Short Paper Proceedings. 2010. Zurich, Switzerland.

53. Therneau TM, Atkinson EJ, Foundation M. An Introduction to Recursive Partitioning Using the RPART Routines 2018 February 23 [cited 2019 February 14th]. https://cran.r-project.org/web/packages/rpart/vignettes/ longintro.pdf.

54. Milborrow S. Plotting rpart trees with the rpart.plot package. 2018 [cited 2019 February 14th]. http://www.milbo.org/rpart-plot/prp.pdf.

55. Gu Z, Eils R, Schlesner M. Complex heatmaps reveal patterns and correlations in multidimensional genomic data. Bioinformatics. 2016;32(18):2847-9.

56. Lex A, Gehlenborg N, Strobelt H, Vuillemot R, Pfister H. UpSet: visualization of Intersecting Sets. IEEE Trans Visual Comput Graphics. 2014;20(12):1983-92.

57. Sebastien L, Julie J, Francois $H$. FactoMineR: an R package for multivariate analysis. J Stat Softw. 2008;25:1-8.

58. Alboukadel K, Practical Guide to Principal Component Methods in R. 2018.

59. Gabriel KR. The biplot graphic display of matrices with application to principal component analysis. Biometrika. 1971;58(3):453-67.

60. Potgieter A B, George-Jaeggli B, Chapman S C, Laws K, Suárez Cadavid $L$ A, Wixted J, Watson J, Eldridge M, Jordan D R, and Hammer G L, Multispectral imaging from an unmanned aerial vehicle enables the assessment of seasonal leaf area dynamics of sorghum breeding lines. Front Plant Sci. 2017:8(1532).

61. Cooper M, Messina CD, Podlich D, Totir LR, Baumgarten A, Hausmann NJ, Wright D, Graham G. Predicting the future of plant breeding: complementing empirical evaluation with genetic prediction. Crop Pasture Sci. 2014:65(4):311-36

62. Mendes-Moreira P, Alves ML, Satovic Z, Dos Santos JP, Santos JN, Souza JC, Pêgo SE, Hallauer AR, Vaz Patto MC. Genetic architecture of ear fasciation in maize (Zea mays) under QTL scrutiny. PLOS ONE. 2015;10(4):e0124543-e43.

63. Walter A, Liebisch F, Hund A. Plant phenotyping: from bean weighing to image analysis. Plant Methods. 2015;11:14.

64. Dhondt S, Wuyts N, Inze D. Cell to whole-plant phenotyping: the best is yet to come. Trends Plant Sci. 2013;18(8):433-44.

65. Hopkins AA, Saha MC, Wang ZY. Breeding, genetics, and cultivars, in tall fescue for the twenty-first century. Fribourg HA, Hannaway DB, West CP, Editors. 2009, American Society of Agronomy.

66. Crain J, Mondal S, Rutkoski J, Singh RP, Poland J. Combining highthroughput phenotyping and genomic information to increase prediction and selection accuracy in wheat breeding. Plant Genome 2018:11(1):1-14.

\section{Publisher's Note}

Springer Nature remains neutral with regard to jurisdictional claims in published maps and institutional affiliations.

Ready to submit your research? Choose BMC and benefit from

- fast, convenient online submission

- thorough peer review by experienced researchers in your field

- rapid publication on acceptance

- support for research data, including large and complex data types

- gold Open Access which fosters wider collaboration and increased citations

- maximum visibility for your research: over 100M website views per year

At $B M C$, research is always in progress.

Learn more biomedcentral.com/submissions 